\title{
Attenuation of Ground-Motion Spectral Amplitudes and Its Variations across the Central Alborz Mountains
}

\author{
by Khalil Motaghi* and Abdolreza Ghods
}

\begin{abstract}
We selected 136 earthquakes recorded at stations of the Institute of Geophysics of the University of Tehran (IGUT) during 1996-2007, in order to estimate the average attenuation parameters and variations of station corrections across the central Alborz mountains, the northern extension of the Alpine-Himalayan orogeny in western Asia. The selected events provide 1260 high signal-to-noise ratio (SNR) records with relatively good spatial coverage. We graphically find the distances at which the nature of geometrical spreading attenuation $\left(R^{b}\right)$ changes significantly using a locally weighted scatterplot smoothing (LOWESS, local regression smoothing method) called robust LOWESS. A trilinear function with hinges at distances of about 80 and $160 \mathrm{~km}$ describes the geometric spreading attenuation with distance. By regressing to the hinged trilinear function, we found that $b_{1}=-1.15 \pm 0.21, b_{2}=0.09 \pm 0.31$, and a fixed $b_{3}=-0.5$ minimize the average absolute value of the Fourier spectrum amplitude residuals. Using an anelastic attenuation coefficient at different frequencies, the direct quality factor $Q$ in the central Alborz region is obtained as $Q=$ $109 \pm 2 f^{0.64 \pm 0.04}$. The geographical distribution of station corrections at $1 \mathrm{~Hz}$ can be clearly separated along a dividing line connecting the North Tehran, Mosha, and Attari faults into two regions: the northern low-attenuating and the southern high attenuating regions. The stations with strong positive station corrections clearly align along the dividing line. This suggests the existence of a higher attenuation zone south of the dividing line. The presence of thick Tertiary-Quaternary sedimentary rocks with thick layers of salt in the southern region may explain the observed greater attenuation of seismic waves.
\end{abstract}

\section{Introduction}

The central Alborz Mountains of northern Iran (Fig. 1) is a zone of intensely active continental deformation, and it is the most heavily populated region of Iran, including the megacity of Tehran with a population of over 10 million. It is a part of the Arabia-Eurasia collision zone where deformation is characterized by partitioning of strain into rangeparallel left-lateral strike-slip and thrust faults (Jackson et al., 2002; Allen et al., 2003). The Alborz region is affected by numerous active faults, some of which have great seismic potential, and documented historical seismicity (Ambraseys and Melville, 1982; Berberian and Yeats, 1999). As such, the central Alborz Mountains are a prime candidate for seismic hazard evaluation studies

The choice of ground-motion model has a significant impact on hazard estimates for the distance range of 50$200 \mathrm{~km}$ within an active seismic zone such as the central Alborz. Estimation of ground shaking for a typical frequency

\footnotetext{
*Now at the International Institute of Earthquake Engineering and Seismology (IIEES), Tehran, Iran.
}

range of $0.2-10 \mathrm{~Hz}$ is required for the proper design of earthquake resistant structures and facilities (Raoof et al., 1999) and is considered as input for engineering stochastic groundmotion relationships (Atkinson, 2004). The lack of an appropriate ground-motion prediction model may result in undesirable outcomes, such as unrealistically high or low loading standards in the design and construction of critical infrastructure such as large dams, power stations, and hospitals. For seismological purposes, appropriate attenuation models make it possible to calculate more accurately source parameters such as magnitude and seismic moment (Atkinson and Mereu, 1992).

The Alborz region has experienced very few large events during the operation of the accelerometer network of the Building and Housing Research Center (BHRC). The BHRC network has been operating since 1973 but has recorded ground acceleration for only 22 events in the study area, because of the low seismicity rate (Ghods and Sobouti, 2005).

Zare (1999) and Ghasemi et al. (2009) produced ground-motion relationships for Iran using BHRC acceler- 


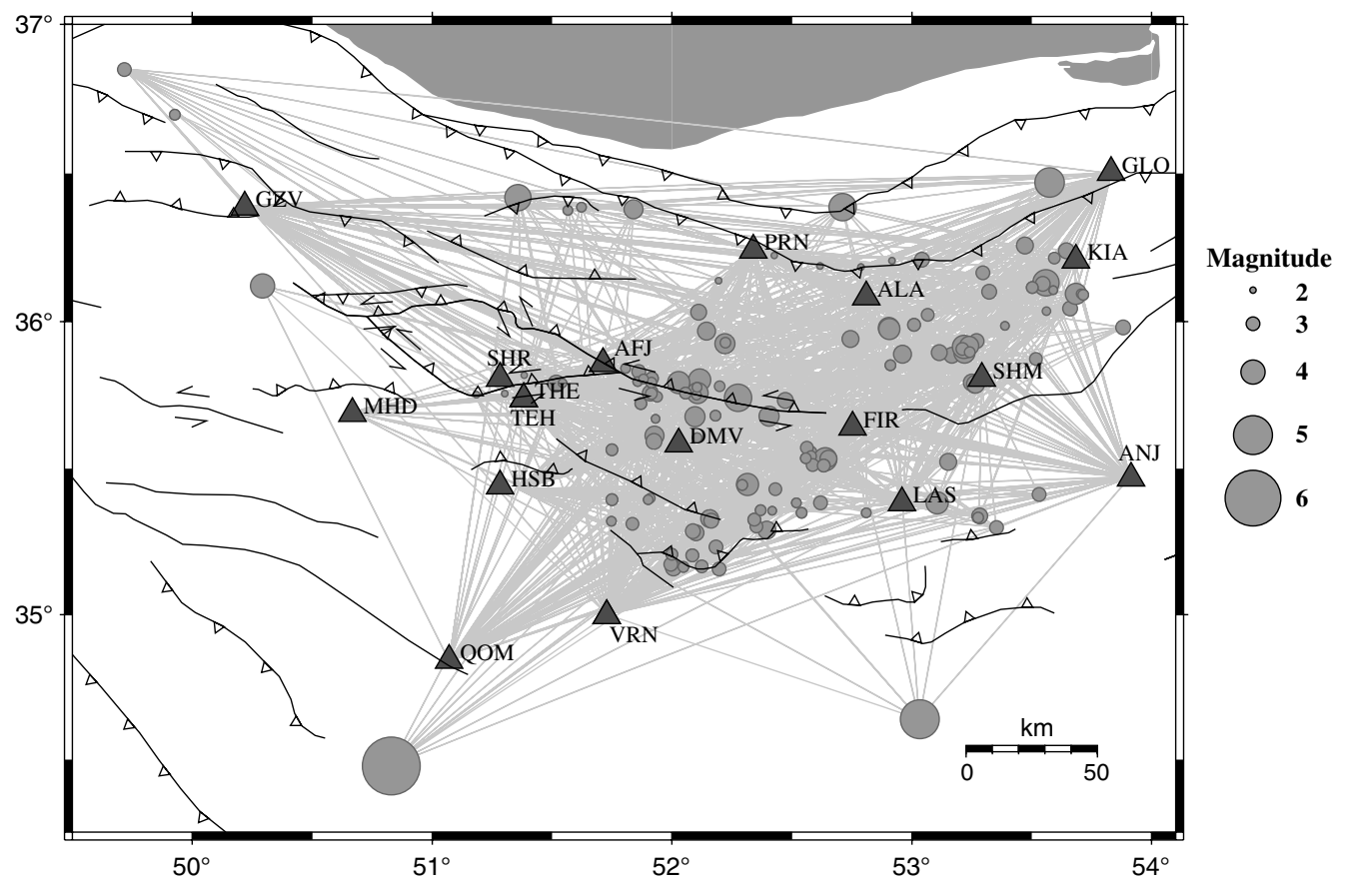

Figure 1. The epicenters of 136 selected events used in this study are shown as circles with sizes related to their local magnitude. The location of IGUT stations are shown as triangles. The fault traces are shown as black lines (Hessami et al., 2003). The ray coverage of the 1260 records used in this study is also shown.

ometer records. The ground-motion relationship is valid for distances up to $100 \mathrm{~km}$ and is derived from events mostly larger than $M_{\mathrm{w}} 5$. Motazedian (2006) calculated the attenuation relationship for northern Iran, including the Alborz region, using 761 acceleration time series recorded by 259 three-component BHRC instruments for 22 large events in the Alborz. A large portion (about 50\%) of the dataset used by Motazedian (2006) belongs to the $2004 M_{\mathrm{w}} 6.2$ BaladehKajour event, making their relationship possibly biased toward the north central Alborz.

It has been shown that the spatial distribution of events and stations may introduce an azimuthal dependency on the amplitude of the ground motion (Campbell and Bozorgnia, 1994). In addition, BHRC stations are usually installed inside governmental buildings and rarely have information concerning the site condition. The records are therefore heavily contaminated by site effects, leading to increased scatter in the amplitude readings.

The attenuation relationship derived using large events may not be used directly for smaller seismic sources where the seismic sources can be considered as point sources. The geometrical spreading coefficients of around -0.5 obtained by Zare and Sabzali (2006) and Motazedian (2006) are only applicable for large shallow events during which seismic waves spread as nearly cylindrical waves. It has been shown that the attenuation relationship derived from large events often overpredicts the ground motion for smaller events (Bommer et al., 2007)

In this work, we derive a relationship for attenuation of ground-motion spectral amplitudes that is strictly valid for point-source events and for the magnitude range considered in this study. This relationship is appropriate for seismological studies such as moment tensor inversion. Using our attenuation relationship, one can calculate the attenuation relationship for larger events using finite fault modeling (Hartzell, 1978) and then assess whether the attenuation relationship is good for larger events. A ground-motion prediction equation (GMPE) for the study region can be calculated using the attenuation relationship developed in this study. GMPEs predict 5\% damped response spectral acceleration for the Fourier acceleration models, including those provided by attenuation relationships. Boore (2003) gave a comprehensive description of the methods to calculate GMPEs from attenuation relationships.

The availability of the abundant weak-motion waveform data from the short-period local seismograph network of the Institute of Geophysics of the University of Tehran (IGUT) provides an opportunity to derive a new and more reliable ground-motion relationship for small events to complement those of strong-motion results. Several recent works have used a similar approach to derive attenuation relationship from weak-motion data (e.g., Allen et al., 2007; Massa et al., 2008; Meirova et al., 2008). We have selected 136 events ranging between $M_{\mathrm{L}} 2.1$ and 6.1. The distribution of magnitudes of the selected events is heavily skewed toward small magnitude events. This implies that our ground-motion relationship cannot be directly extrapolated for larger events outside of the magnitude range of those in our catalog. Despite this shortcoming, this study provides a reliable groundmotion relationship for a distance range of $20-250 \mathrm{~km}$ 
and could set the stage for further development of a regional ground-motion relationship for the central Alborz area.

Our study may allow for more effective separation of source and station effects from path effects once compared with the BHRC accelorometric dataset. This is because most of the stations and events used in our study have larger numbers of rays coming from different azimuths (Fig. 1). For the existing accelerometer dataset, the source and station terms cannot be estimated because the dataset is dominated by one large event. Bommer et al. (2007) showed that the GMPEs exhibit a strong dependence on magnitude but a very low dependence on geometrical spreading. Thus, our approach of using weak-motion data gives us the opportunity to provide a reliable geometrical spreading relationship for small magnitude events.

We also calculate the direct quality factor for the region and compare it with the coda quality factor for the region recently calculated by Rahimi et al. (2010). Furthermore, we determine station corrections (i.e., the log of the observed Fourier amplitudes minus the log of the predicted Fourier amplitudes) and relate its geographical variations to the thickness and type of sedimentary layers in the region.

\section{Data}

The analysis presented in this article is based on data collected by the IGUT. Founded in 1995 by the University of Tehran, IGUT installed the first modern digital seismic network in Iran. The network is made up of several provincial subnetworks. Ghods and Sobouti (2005) gave a detailed description of the hardware and the network setup of IGUT. Each station is equipped with three-component shortperiod SS-1 seismometers (made by Kinemetrics) with an eigenfrequency of $1 \mathrm{~Hz}$ and 24-bit digitizer with a sampling rate of 50 samples per second. Each subnetwork is equipped with a central Global Positioning System (GPS) receiver for timing.

Our study area encompasses the central Alborz range and the plains to the south that are part of the northern margins of the central Iran block (Fig. 1). The three IGUT subnetworks of Mazandaran, Semnan, and Tehran cover this area. The average interstation distance inside the central Alborz region is about $50 \mathrm{~km}$; and except for station KIA, all stations used in this study are installed on a solid rock basement. Table 1 lists the latitudes, longitudes, and elevations of the stations used in the study.

Figure 1 shows the locations of the seismic stations and the 136 selected events. We used only events recorded by more than three stations and relocated the events using all phases from all the subnetworks. For those events that lie closer to the corners of the study area, we used phase readings from neighboring subnetworks to the south. The biased epicentral location error for the events is less than $5 \mathrm{~km}$. The hypocentral depth of the events has poor accuracy. Most of the events have focal depths close to $15 \mathrm{~km}$, which is in agreement with the pattern of seismicity in the region (Bondar et al., 2004). The Alborz is a zone of compressional deformation, and seismicity is mostly limited to the upper $30 \mathrm{~km}$ of the crust.

The selected events mostly lie inside the central Alborz. This ensures that the events belong to one tectonic zone and have a rather uniform stress drop range. Figure 1 shows that the selected events provide relatively homogenous ray coverage inside the study area. The magnitudes of the 136 selected events range from $M_{\mathrm{L}} 2.1$ to 6.1 , as calculated from the

Table 1

Station Information and Station Correction Values for $1 \mathrm{~Hz}^{*}$

\begin{tabular}{cccccccc}
\hline Station Code & Latitude $\left({ }^{\circ} \mathrm{N}\right)$ & Longitude $\left({ }^{\circ} \mathrm{E}\right)$ & Altitude $(\mathrm{m})$ & Station Correction & Number of Records & std & Station Correction $R<100 \mathrm{~km}$ \\
\hline AFJ & 35.8560 & 51.7125 & 2750 & 0.12 & 61 & 0.22 & 0.13 \\
ALA & 36.0829 & 52.8099 & 2600 & 0.24 & 49 & 0.30 & 0.25 \\
ANJ & 35.4672 & 53.9144 & 2135 & -0.23 & 62 & 0.27 & -0.26 \\
DMV & 35.5772 & 52.0322 & 2546 & -0.06 & 98 & 0.28 & -0.06 \\
FIR & 35.6415 & 52.7536 & 2380 & -0.07 & 99 & 0.27 & -0.08 \\
GLO & 36.5024 & 53.8302 & 1963 & 0.51 & 83 & 0.21 & 0.53 \\
GZV & 36.3859 & 50.2184 & 2100 & -0.02 & 56 & 0.22 & -0.22 \\
HSB & 35.4275 & 51.3567 & 1098 & -0.21 & 67 & 0.17 & -0.11 \\
KIA & 36.2070 & 53.6837 & 2167 & -0.10 & 58 & 0.28 & -0.13 \\
LAS & 35.3822 & 52.9589 & 2195 & -0.17 & 77 & 0.18 & - \\
MHD & 35.6853 & 50.6675 & 1150 & -0.21 & 27 & 0.32 & 0.10 \\
PRN & 36.2419 & 52.3381 & 1333 & 0.12 & 81 & 0.26 & 0.08 \\
QOM & 34.8424 & 51.0703 & 2270 & -0.13 & 69 & 0.23 & 0.10 \\
SHM & 35.8067 & 53.2922 & 2500 & 0.11 & 79 & 0.29 & 0.27 \\
SHR & 35.806 & 51.283 & 1470 & 0.09 & 34 & 0.18 & -0.22 \\
TEH & 35.7367 & 51.3817 & 1462 & 0.28 & 26 & 0.24 & 0.23 \\
VRN & 34.9954 & 51.7273 & 855 & -0.18 & 118 & & \\
\hline
\end{tabular}

*The number of records used to calculate the station corrections and the standard deviation $(s t d)$ of the Fourier spectral amplitude residuals are given in the sixth and seventh columns, respectively. Station corrections calculated using only rays with hypocentral distances less than $100 \mathrm{~km}$ are given in the last column. 


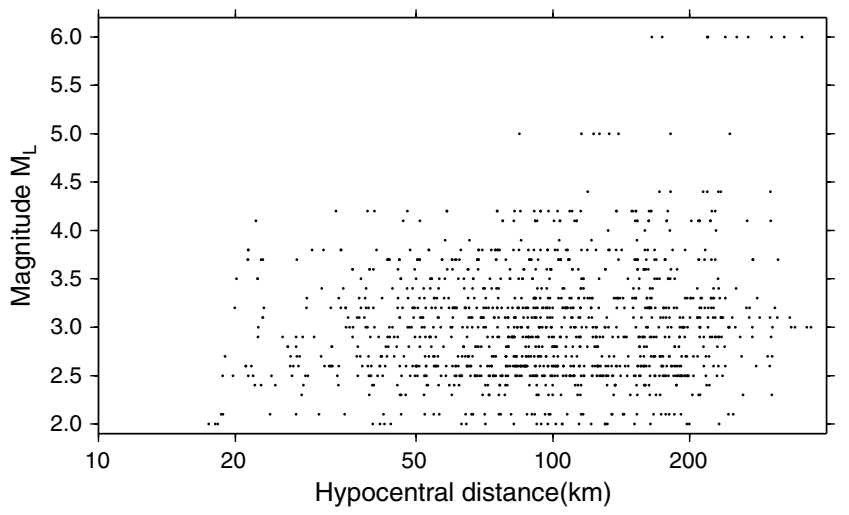

Figure 2. Magnitude $\left(M_{\mathrm{L}}\right)$ distribution of selected records versus hypocentral distance.

Askari et al. (2009) $M_{\mathrm{L}}$ relationship. Figure 2 shows the distribution of magnitudes versus hypocentral distances.

In this study, we used 1260 velocity records of the 136 events recorded by 17 IGUT seismic stations in the period 1996-2007. The selected waveforms are unclipped and have high signal-to-noise (SNR) ratio. The records used in the regression analysis belong to IGUT stations lying inside or in the near vicinity of the central Albroz (Table 1).

Figure 3 displays the distribution of the selected records for each station as a function of hypocentral distance. We note that the spatial sampling of the selected records is fairly good and well distributed for hypocentral distances ranges of 20-250 km. The selected source-receiver distances are equal to or larger than $20 \mathrm{~km}$. Due to significant uncertainty in hypocentral distances, we limited our regression analysis to hypocentral distances equal to or greater than $20 \mathrm{~km}$.

We did not include accelerometer amplitude readings in our dataset because their site response in most cases is not

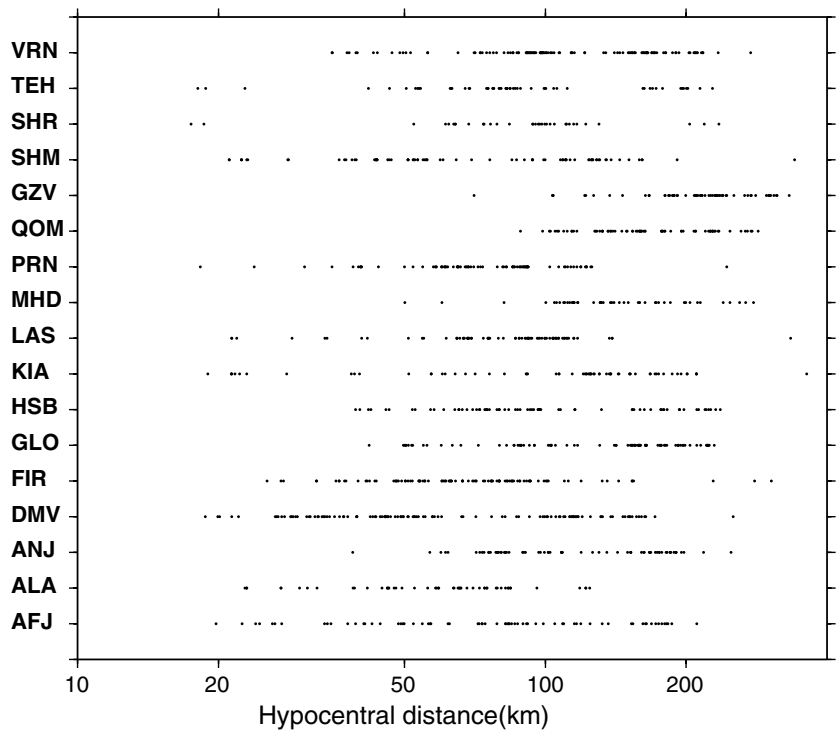

Figure 3. Distribution of the selected records versus hypocentral distance and station. known. As shown by Steidl et al. (1996), Boore et al. (1997), and Lussou et al. (2001) differences in site conditions strongly affect the amplitude and frequency content of strong motions. Motazedian (2006) has already derived attenuation parameters for our study region using BHRC data, but his dataset is strongly biased in terms of event sampling of the region. Many of the records (about 50\%) used in his analysis belong to a single event, the $2004 M_{\mathrm{w}} 6.2$ Baladeh-Kajour event. Most importantly, we wanted to avoid larger events to make sure that all of our events can be safely considered as simple point sources. This later assumption helps us to provide attenuation parameters for strictly point-like sources. Using the attenuation relationship derived in this study, one can calculate the attenuation relationship for larger events using finite fault modeling (Hartzell, 1978).

\section{Signal Processing}

The amplitude spectra were calculated following the procedure presented by Boore (2010). We combined the two north-south and east-west seismograms into a single seismogram for a given azimuth using equation 1 of Boore (2010). We used rotation angles with a $1^{\circ}$ increment of rotation with a range from $0^{\circ}$ to $179^{\circ}$. For each period, we calculated the amplitudes for the 180 rotation angles and then took the median as the amplitude for the given period. The horizontal amplitudes are not significantly affected by site specifications because the seismic stations used in this study are mostly on hard rock (Ghods and Sobouti, 2005). For the calculation of amplitude spectrum at a given rotation angle, we followed the method of Atkinson and Mereu (1992). For each rotated combined horizontal record, a shear-wave window was selected and a 5\% taper was applied at each end of the window. The shear-wave window contains $90 \%$ of the shear-wave energy and includes direct, reflected, and refracted shear phases. After correcting for instrument response, the Fourier spectrum of the shear-wave window and a noise window with the same length as the shear-wave window were calculated and binned in increments of $0.1 \log$ frequency units for a central frequency range of $0.63-12.56 \mathrm{~Hz}$.

Only those Fourier spectrum amplitudes for which SNR exceeded two were used in our regression analysis. Our SNR criterion was applied for each frequency, not for each record. The Fourier amplitudes used in the regression analysis were corrected for noise amplitude by subtracting the corresponding smoothed binned noise amplitude from the observed signal amplitude. We note that the compiled spectral data are not affected by low-amplitude quantization problems.

\section{Ground-Motion Model}

We fit the observed Fourier velocity amplitudes $(\mathrm{m} / \mathrm{sec})$ at each frequency $A_{i j}(f)$ to the following equation:

$$
\log A_{i j}(f)=a_{1}(f)+a_{2}(f) M_{i}+b(f) \log R_{i j}+c(f) R_{i j},
$$


where $M_{i}$ is the moment magnitude $M_{\mathrm{w}}$ of an event $i$ as calculated using attenuation parameters derived in the present study, $R_{i j}$ is the hypocentral distance for the $i$-th event and $j$-th station, and $a_{1}, a_{2}, b$, and $c$ are constant parameters that should be determined for each frequency $f$. Parameters $a_{1}$ and $a_{2}$ take into account the dependence of $A_{i j}(f)$ on the source effect. The coefficients $b$ and $c$ are related to geometrical spreading and anelastic attenuation, respectively. No station correction term is considered in equation (1). The station corrections are computed later based on the analysis of our regression residuals.

In our regression formula, magnitude $M$ stands for moment magnitude $M_{\mathrm{w}}$. We initially regressed equation (1) against local magnitude $M_{\mathrm{L}}$ to calculate the attenuation parameters. Moment magnitudes were then calculated by using the distance-corrected low-frequency asymptote of the Fourier displacement spectrum of all records and then averaged for each event. We calculated $M_{\mathrm{w}}$ for all of our events, excluding the largest $M_{\mathrm{L}} 6.1$ event. Adding the reported

2 HRV $M_{\mathrm{w}} 5.7$ magnitude for the largest event into our dataset, we could calculate the following relationship between $M_{\mathrm{w}}$ and $M_{\mathrm{L}}$ (Fig. 4):

$$
M_{\mathrm{w}}=(0.74 \pm 0.05) M_{\mathrm{L}}+(0.72 \pm 0.14)
$$

Many ground-motion attenuation relationships define the distance parameter to be the shortest distance between the station and the presumed seismogenic rupture (e.g., Campbell, 1997). The majority of the events used in our catalog have relatively low magnitudes and, consequently, small rupture dimensions. This allows us to use a point-source approximation and assume $R_{i j}$ to be the hypocentral distance.

In a layered crustal model, different phases arrive at different distances, distorting the monotonic amplitude decay

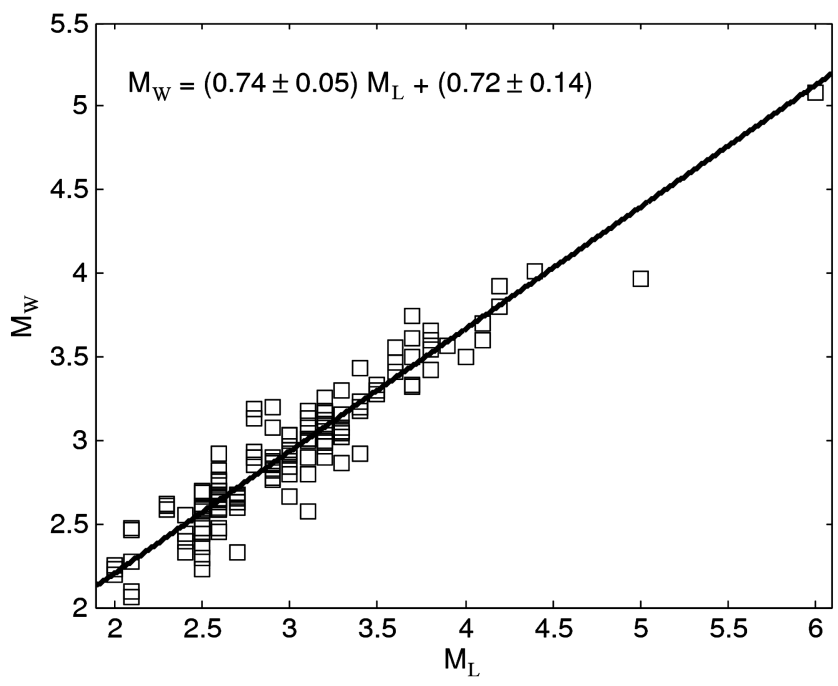

Figure 4. Relationship between $M_{\mathrm{L}}$ (Askari et al., 2009) and $M_{\mathrm{w}}$. A systematic difference exists between the two magnitude scales. with the distance of a half-space medium. To include the effect of layered media on the geometrical spreading into our regression analysis, we allow $b$ to take different values at different distances.

In eastern North America, for hypocentral distances up to $70 \mathrm{~km}$, the shear window is dominated by the direct $S$-wave arrival (e.g., Burger et al., 1987; Atkinson, 2004). The arrival of postcritical reflections from the Conrad and Mohorovičić discontinuities join with the direct $S$ arrival within the distance range of 70-120 km (Burger et al., 1987). The $L g$ phase starts at distances beyond $100-150 \mathrm{~km}$ (e.g., Ou and Hermann, 1990). Atkinson and Mereu (1992) and Atkinson and Boore (1995) modeled the attenuation using a hinged trilinear function that has three different values of $b$ for its three segments.

The anelastic coefficient $c(f)$ is related to the direct quality factor $Q$ as follows (Atkinson and Mereu, 1992):

$$
Q(f)=\frac{\pi f}{\ln (10) c(f) \beta},
$$

where $\beta$ is the shear-wave velocity and $Q$ describes the combined intrinsic and scattering attenuation of the direct shear seismic waves.

Except for a few technicalities, the regression analysis presented in this study closely follows that of Atkinson and Mereu (1992), who performed their fitting assuming different values for the hinge points. Instead, we graphically found the optimal hinge points for each frequency by using a magnitude-stacked function of amplitude versus distance. To find a magnitude-stacked function of amplitude $A_{i j}^{\prime}(f)$ versus distance, we tried to remove the source effect by finding the best fit to the following equation using the least squares method:

$$
\begin{aligned}
& \log A_{i j}(f)+\log R_{i j}=a_{1}(f)+a_{2}(f) M_{i} \\
& \quad \text { for all } i \text { with } R_{i j} \leq 100 \mathrm{~km} .
\end{aligned}
$$

The geometrical spreading coefficient was assumed to be -1 , as theory suggests for body waves spreading in a homogenous half-space. In equation (4), anelastic attenuation and scattering were assumed to be negligible for the frequency range studied here and for $R_{i j} \leq 100 \mathrm{~km}$. Using the calculated $a_{2}$ coefficient, $\log A_{i j}^{\prime}(f)$ was calculated using $\log A_{i j}^{\prime}(f)=\log A_{i j}-a_{2} M_{i}$. The calculated $a_{1}$ and $a_{2}$ coefficients were only used to stack amplitudes for graphical purposes and later recalculated using a one-step regression method. We note that anelastic attenuation and scattering are only negligible for the lower end of the frequency range studied here. However, plotting $A_{i j}^{\prime}(f)$ versus hypocentral distance for all frequencies studied here shows a strong consistency in the number and location of the detected hinge points across all frequencies. This implies that the variation of attenuation with frequency does not change the location and number of hinge points. The calculations of $a_{1}$ and $a_{2}$ are 
done for each frequency, so constraining equation (4) to $f<$ $2 \mathrm{~Hz}$ does not have any effect on the hinge points.

Figure 5 shows that the logarithm of Fourier spectral amplitudes corrected for geometrical spreading effects (i.e., $\left.\log A_{i j}(f)+\log R_{i j}\right)$ at $1.58 \mathrm{~Hz}$ has the following linear relationship with moment magnitude:

$$
\log A_{i j}(f)+\log R_{i j}=(1.5 \pm 0.08) M_{\mathrm{w}}-(6.32 \pm 0.25) .
$$

This relationship was calculated by the least squares method. The linear relationship was also obtained for other frequencies studied in this work. The constant 1.5 in equation (5) is fully consistent with the definition of $M_{\mathrm{w}}$ (Kanamori, 1977; Hanks and Kanamori, 1979). Figure 6 compares the Fourier spectra of amplitudes at $1.58 \mathrm{~Hz}$ (top panel) with those of magnitude-stacked Fourier spectral amplitudes (bottom panel). The magnitude-stacked amplitudes show significantly smaller scatter.

To graphically find the best hinge points for the geometrical spreading function, we used a locally weighted scatterplot smoothing (LOWESS) algorithm called robust LOWESS (Cleveland, 1979; Cleveland et al., 1992). The method uses locally weighted linear regression to smooth the data. The regression method uses a linear polynomial while using a local smoothing process. The smoothing process is local because it calculates each smoothed value by a set of neighboring data points. The smoothing process is also weighted because a regression weight function is defined for the data points contained within the data spans. In addition to the regression weight function, the method uses a robust weight function to make the process resistant to outliers. Using the LOWESS method, we fitted a smoothed curve onto the log of magnitude-stacked amplitudes $\log A_{i j}^{\prime}(f)$ (Fig. 7). We detected hinges in the attenuation curves at $\sim 80, \sim 120$, and

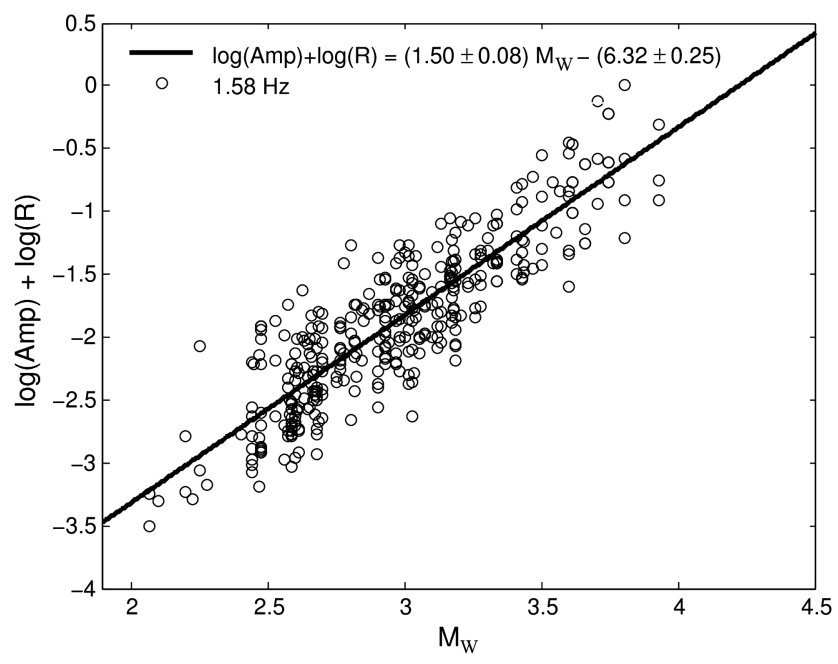

Figure 5. Fourier spectral amplitude corrected for geometrical spreading (hollow circles) versus local magnitude for a frequency of $1.58 \mathrm{~Hz}$. The solid line is the fitted line using equation (4).
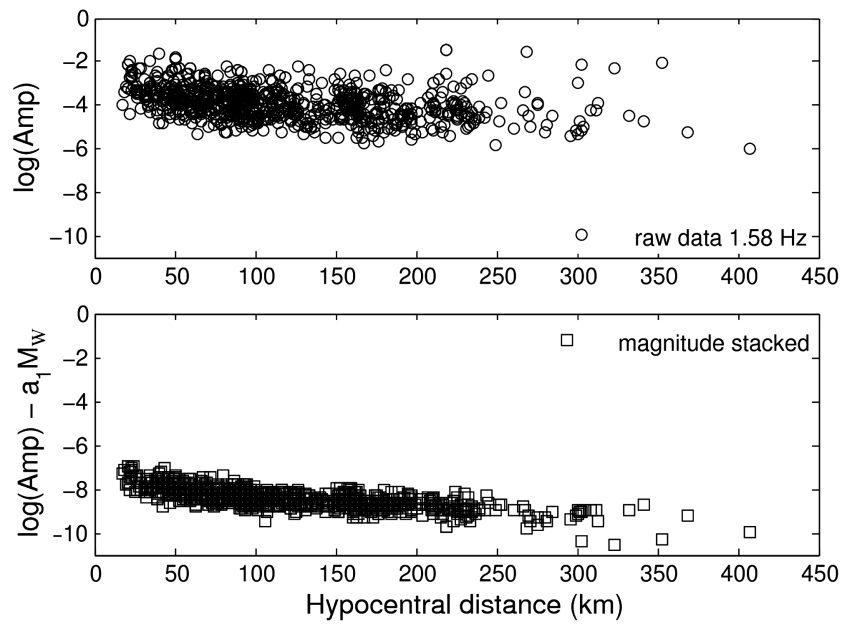

Figure 6. Fourier spectral amplitude (top panel) and magnitude-stacked Fourier spectral amplitude (bottom panel) versus distance for a frequency $1.58 \mathrm{~Hz}$. The vertical axes in both subplots have the same scale to illustrate scattering of the dataset before and after stacking.

$\sim 160 \mathrm{~km}$ (Fig. 7). These hinges are recognizable at all frequencies as shown for 1.58, 3.15, 3.97, 5.0, and $7.92 \mathrm{~Hz}$.

The segment between the first and third hinges is the most important flattening in the attenuation curve. Our synthetic tests show that inclusion of all three hinges in our regression reduces the number of data points for the intervals 3 of $80-120 \mathrm{~km}$ and $120-160 \mathrm{~km}$, and thus makes it impossible to resolve reliably the assumed initial coefficients. Therefore, we include only the hinges at 80 and $160 \mathrm{~km}$ in our ground-motion model. Beyond $80 \mathrm{~km}$, the presence of strong postcritical reflections from the Conrad and

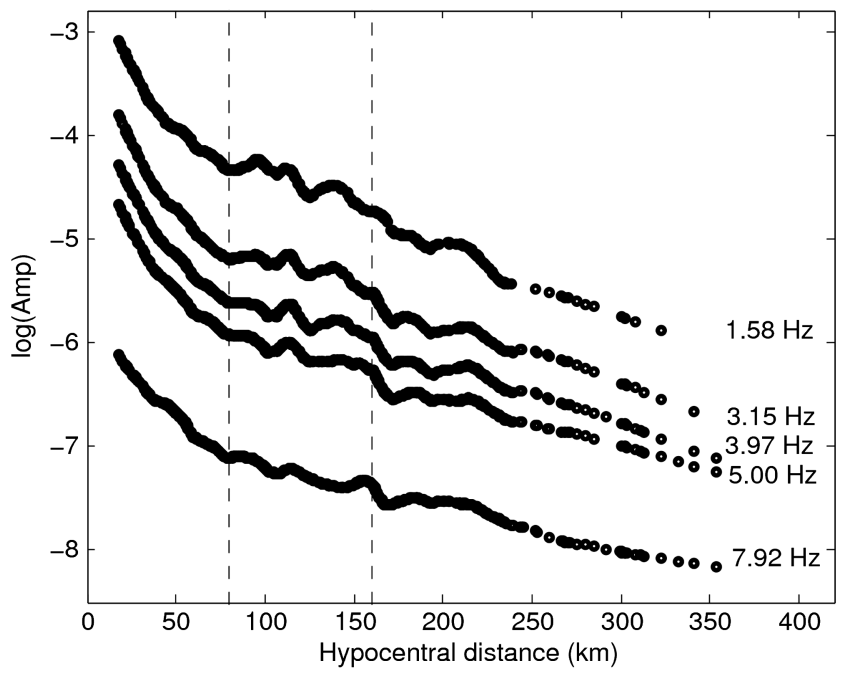

Figure 7. The best-fit curves for variations of magnitudestacked Fourier spectral amplitudes versus distance for different frequencies. We used a smoothing algorithm called Robust LOWESS to find the best-fit curves. The dashed lines show a region $(80-160 \mathrm{~km})$ in which the slope of the curves is nearly zero. This region is related to postcritical reflections from the Mohorovičić discontinuity. 
Mohorovičić discontinuities produces the flattening of the attenuation curve (Burger et al., 1987; Atkinson, 2004; Motazedian, 2006), leading to little apparent attenuation between approximately 80 and $160 \mathrm{~km}$.

Using a synthetic dataset, we found that the quasiNewtonian method (Fletcher, 1972) could successfully resolve the assumed coefficients for equation (1). Using equation (6) and the coefficients listed in Table 2, we produced a synthetic amplitude dataset for the records in our observed dataset for a frequency of $1.58 \mathrm{~Hz}$. To test the resolving power of our regression method as realistically as possible, the coefficients listed in Table 2 were chosen to closely follow those calculated later by regressing equation (6) to the observed data (Table 3).

To model the usual random scattering in amplitude data, we added normally distributed random numbers to the synthetic amplitudes in such a way that the standard deviation std for the synthetic amplitude residuals becomes 0.35 . This std is the average value for the standard deviation of residuals in our observed dataset. We generated 1000 realizations of synthetic datasets and estimated the coefficients $a_{1}, a_{2}, c$, $b_{1}, b_{2}$, and $b_{3}$ using our one-step regression method.

To avoid local minima while using the quasi-Newtonian method, we used starting point combinations with ranges of $b_{1}$ from -1.0 to $-1.4, b_{2}$ from -0.4 to +0.4 , and $b_{3}$ from -0.1 to -1.0 . For each parameter, a step of 0.1 was used. Starting points for the anelastic attenuation coefficient $c$ were $-0.003,-0.002,-0.001$, and -0.0005 . This numerical experiment showed that the method calculates close estimates for $a_{1}, a_{2}, c, b_{1}$, and $b_{2}$ but not for $b_{3}$. Therefore, we set $b_{3}$ to its theoretical value of -0.5 . The calculated stds of $a_{1}, a_{2}, c$, $b_{1}$, and $b_{2}$ are $0.16,0.02,0.0004,0.09$, and 0.29, respectively. Because of a similar std for the synthetic and observed datasets of the Fourier spectrum amplitudes, the std values calculated for the coefficients using the synthetic data could be considered as the std of the coefficients obtained from real dataset.

We assumed that $b$ has a trilinear shape with hinges at $R_{1}=80 \mathrm{~km}$ and $R_{2}=160 \mathrm{~km}$. Thus, we rewrite equation (1) as follows:

$$
\begin{aligned}
& \log A_{i j}=a_{1}(f)+a_{2}(f) M_{i}+b_{1}(f) \log R_{i j}+c(f) R_{i j} \\
& \quad \text { for } R \leq 80 \mathrm{~km},
\end{aligned}
$$

$$
\log A_{i j}=a_{1}(f) M_{i}+b_{1} \log R_{1}+b_{2} \log \left(\frac{R_{i j}}{R_{1}}\right)+c(f) R_{i j}
$$

for $80 \leq R \leq 160 \mathrm{~km}$, and

Table 2

Attenuation Parameters Used to Produce the Synthetic Data

\begin{tabular}{cccccc}
\hline$a_{1}$ & $a_{2}$ & $b_{1}$ & $b_{2}$ & $b_{3}$ & $c$ \\
\hline-5.59 & 1.38 & -1.15 & +0.09 & -0.5 & -0.0030 \\
\hline
\end{tabular}

Table 3

Attenuation Coefficients Calculated Using a One-Step Regression Method*

\begin{tabular}{rcccrcc}
\hline$f$ & $a_{1}$ & $a_{2}$ & \multicolumn{1}{c}{$b_{1}$} & \multicolumn{1}{c}{$b_{2}$} & $c$ & $s t d$ \\
\hline 0.63 & -6.10 & 1.38 & -1.00 & -0.25 & -0.0014 & 0.33 \\
0.79 & -6.01 & 1.38 & -1.02 & -0.35 & -0.0016 & 0.32 \\
1.00 & -5.69 & 1.39 & -1.16 & -0.39 & -0.0020 & 0.32 \\
1.26 & -5.59 & 1.38 & -1.15 & -0.04 & -0.0030 & 0.30 \\
1.58 & -5.42 & 1.36 & -1.16 & -0.10 & -0.0032 & 0.30 \\
1.99 & -5.03 & 1.32 & -1.30 & 0.07 & -0.0036 & 0.31 \\
2.51 & -4.58 & 1.27 & -1.42 & 0.09 & -0.0039 & 0.31 \\
3.15 & -4.39 & 1.20 & -1.40 & -0.08 & -0.0042 & 0.31 \\
3.97 & -4.21 & 1.16 & -1.41 & 0.20 & -0.0052 & 0.32 \\
5.00 & -4.10 & 1.06 & -1.31 & 0.40 & -0.0062 & 0.34 \\
6.29 & -4.47 & 1.04 & -1.12 & 0.12 & -0.0063 & 0.40 \\
7.92 & -5.06 & 1.02 & -0.75 & 0.49 & -0.0085 & 0.41 \\
9.98 & -4.74 & 0.93 & -0.89 & 0.53 & -0.0086 & 0.40 \\
12.56 & -4.50 & 0.82 & -0.97 & 0.52 & -0.0088 & 0.41
\end{tabular}

*The first column is the central frequency $f$. Hinge points were assumed to be at 80 and $160 \mathrm{~km}$. The standard deviation (std) of amplitude residuals for each central frequency is given in the last column.

$$
\begin{aligned}
\log A_{i j}= & a_{1}(f)+a_{2}(f) M_{i}+b_{1} \log R_{1}+b_{2} \log \left(\frac{R_{2}}{R_{1}}\right) \\
& -0.5 \log \left(\frac{R_{i j}}{R_{2}}\right)+c(f) R_{i j}
\end{aligned}
$$

for $R>160 \mathrm{~km}$.

Table 3 shows the results of our one-step regression analysis on amplitudes using the formulas in equation (6). The standard deviation of amplitude residuals for each frequency is given in the last column of Table 3. The residual is defined as the $\log$ of the observed Fourier amplitudes minus the $\log$ of the Fourier amplitudes predicted by equation (6). These std values varied between 0.30 and 0.41 .

We found that the absolute value of $b_{1}$ for the frequency range of $0.79-6.29 \mathrm{~Hz}$ is above one, thus indicating superspherical geometrical spreading. This behavior has been observed by many others (e.g., Atkinson, 2004) and is attributed partially to the reflection of up-going $S$ waves at the base of the layer above the focus (Frankel, 1991) and partially to scattering (Gagnepain-Beyneix, 1987). Assuming that geometrical spreading coefficients are frequency independent, $b_{1}$ and $b_{2}$ were calculated by taking the average of all $b_{1}$ and $b_{2}$ values for different frequencies. The average values of $b_{1}$ and $b_{2}$ are $-1.15 \pm 0.21$ and $0.09 \pm 0.31$, respectively, while $b_{3}$ is fixed at -0.5 . Consistency of the std for the geometrical spreading coefficients, as calculated from the observed data with those std values obtained from synthetic datasets, confirms that geometrical spreading parameters can be considered to be frequency independent.

The positive geometrical spreading coefficient for the second segment $b_{2}$ is an interesting observation for seismic hazard assessment. A positive geometrical spreading coefficient for the distance range $80-160 \mathrm{~km}$ is related to a strong postcritical reflection effect from the Mohorovičić 
discontinuity. Atkinson and Mereu (1992), Atkinson (2004), and Motazedian (2006) have reported a similar behavior.

\section{Quality Factor}

Seismic waves propagating through the Earth are attenuated with distance at rates greater than predicted by geometrical spreading. The contributing factors to remnant attenuation are intrinsic attenuation due to the medium anelasticity and scattering associated with path inhomogeneities (e.g., Dainty, 1981).

The seismic quality factor $Q(f)$ is an intrinsic property of the material that quantifies the loss of wave amplitude with frequency and distance for all processes other than geometrical spreading (Wilkie and Gibson, 1994). To calculate the combined anelastic-scattering attenuation coefficient $c$, we rewrite equation (6) in the following form where we assume that geometrical spreading coefficients are frequency independent:

$$
\begin{aligned}
& \log A_{i j}=a_{1}(f)+a_{2}(f) M_{i}-1.15 \log R_{i j}+c(f) R_{i j} \\
& \quad \text { for } R \leq 80 \mathrm{~km},
\end{aligned}
$$

$$
\begin{aligned}
\log A_{i j}= & a_{1}(f)+a_{2}(f) M_{i}-1.15 \log R_{1}+0.09 \log \left(\frac{R_{i j}}{R_{1}}\right) \\
& +c(f) R_{i j}
\end{aligned}
$$

for $80 \leq R \leq 160 \mathrm{~km}$, and

$$
\begin{aligned}
\log A_{i j}= & a_{1}(f)+a_{2}(f) M_{i}-1.15 \log R_{1}+0.09 \log \left(\frac{R_{2}}{R_{1}}\right) \\
& -0.5 \log \left(\frac{R_{i j}}{R_{2}}\right)+c(f) R_{i j}
\end{aligned}
$$

for $R>160 \mathrm{~km}$.

Equation (7) minimizes the possible trade-off between the combined anelastic-scattering and geometric spreading coefficients in the regression procedure. Using equation (3) and assuming a shear velocity $\beta$ of $3.7 \mathrm{~km} / \mathrm{s}$, the $Q$ values were calculated using the calculated $c(f)$ coefficients (Table 4). Figure 8 shows the calculated $Q$ values. The behavior of the $Q$ values was represented by a U-shaped curve as follows:

$$
\begin{aligned}
\log Q= & (0.18 \pm 0.07)(\log f)^{2}+(0.44 \pm 0.07)(\log f) \\
& +(2.07 \pm 0.02)
\end{aligned}
$$

The U-shaped behavior of the $Q$ value has been observed frequently (e.g., Aki, 1980; Cormier, 1982; Boore, 2003; Atkinson, 2004; Allen et al., 2007). The U-shaped curve of the $Q$ value was approximated for frequencies above 1.0 Hz by a straight line, as shown in Figure 8 . The straight
Table 4

Revised Attenuation Coefficients*

\begin{tabular}{cccccc}
\hline$f$ & $a_{1}$ & $a_{2}$ & $c$ & $s t d$ & $Q$ \\
\hline 0.63 & -5.87 & 1.39 & -0.0018 & 0.33 & 103 \\
0.79 & -5.88 & 1.42 & -0.0023 & 0.32 & 100 \\
1.00 & -5.80 & 1.44 & -0.0029 & 0.32 & 114 \\
1.26 & -5.71 & 1.42 & -0.0032 & 0.30 & 128 \\
1.58 & -5.30 & 1.32 & -0.0036 & 0.30 & 150 \\
1.99 & -5.34 & 1.34 & -0.0039 & 0.31 & 170 \\
2.51 & -5.11 & 1.29 & -0.0043 & 0.31 & 190 \\
3.15 & -4.83 & 1.21 & -0.0049 & 0.31 & 219 \\
3.97 & -4.66 & 1.15 & -0.0053 & 0.32 & 253 \\
5.00 & -4.58 & 1.12 & -0.0058 & 0.33 & 296 \\
6.29 & -4.36 & 1.03 & -0.0062 & 0.39 & 327 \\
7.92 & -4.46 & 1.03 & -0.0071 & 0.41 & 392 \\
9.98 & -4.42 & 0.95 & -0.0075 & 0.40 & 474 \\
12.56 & -4.13 & 0.79 & -0.0078 & 0.40 & 619 \\
\hline
\end{tabular}

*The attenuation coefficients are calculated while fixing the geometrical spreading coefficients (equation 7). The first column is the central frequency $f$. Hinge points were assumed to be at 80 and $160 \mathrm{~km}$. The standard deviation of Fourier spectral amplitude residuals (std) for each frequency and quality factor $Q$ are given in the two rightmost columns, respectively.

line presented in Figure 8 was calculated using a least squares method and is given by

$$
Q=109 \pm 2 f^{0.64 \pm 0.04} \text {. }
$$

The computed $Q_{0}$ value, namely the $Q$ value at $1 \mathrm{~Hz}$, is in the reported range for seismotectonically active regions (e.g., Rahimi et al., 2010). The calculated direct $Q$ is a mixture of intrinsic and scattering attenuation, where only the later is frequency dependent.

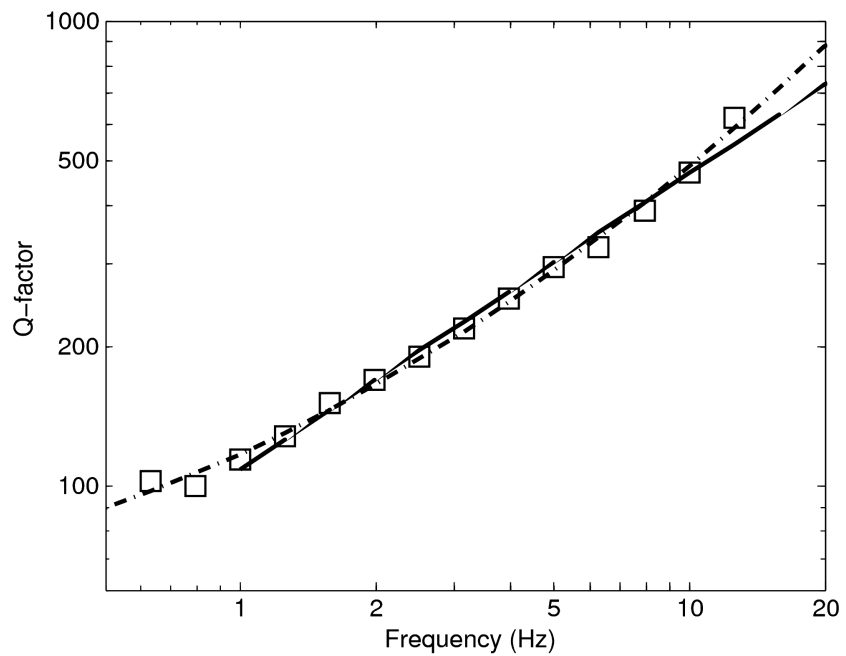

Figure 8. Quality factor (squares) versus frequency, which follows the dashed U-shape curve $\log Q=(0.18 \pm$ $0.07)(\log f)^{2}+(0.44 \pm 0.07)(\log f)+(2.07 \pm 0.02)$. The $Q$ values were fitted by the solid black straight line $\left(Q=109 f^{0.64}\right)$ for frequencies equal to or greater than $1 \mathrm{~Hz}$. 
Rahimi et al. (2010) calculated a coda quality factor for the Alborz region as $Q_{c}=76 \pm 2 f^{1.07 \pm 08}$. The calculated $Q_{c}$ implies that the path attenuation of seismic waves is almost frequency independent, a result that is in contradiction with our results. To calculate $Q_{c}$, we assume a constant geometrical spreading coefficient of one, namely a homogenous half-space, and use a lapse time window of $30 \mathrm{~s}$.

Figure 9 plots the residuals of the regression against different hypocentral distances and magnitude ranges and for two different frequencies of 1 and $5 \mathrm{~Hz}$. There are no discernible trends in the residuals when plotted against the hypocentral distance (the left column panels), confirming that the selected $b$ and $R$ parameters $\left(b_{1}, b_{2}, R_{1}\right.$, and $\left.R_{2}\right)$ are reasonable for the study region. The right column panels in Figure 9 clearly indicate that there are no noticeable trends in the residuals when plotted against magnitude. We conclude that the attenuation model of equation (7) provides a satisfactory description of our spectral amplitudes.

\section{Station Corrections}

The residuals were averaged on a station-by-station basis to determine station corrections. The residual is defined as the log of the observed Fourier amplitude minus the log of the Fourier amplitude predicted by equation (7). The residuals and station corrections were calculated for the frequency range $0.68-12.56 \mathrm{~Hz}$. A large average residual for a particular station would be indicative of a significant site response. Alternatively, the amplitude residuals can be related to the lateral variations of attenuation. A positive residual for a given station is either related to the amplifying effect of the geology beneath the station (i.e., site effects) or to the smaller attenuation along the paths of seismic waves to the station (i.e., path effects). As shown in Figure 9, the standard deviation of the residuals tend to be much larger than the average residual values. Thus, one cannot significantly reduce data variability and scatter using the station corrections.

Figure 10 shows the spatial distribution of station corrections for $1 \mathrm{~Hz}$. The distribution of positive and negative station corrections are nearly divided into two regions (northern and southern regions) along the North Tehran-MoshaAttari faults (Fig. 10), hereafter called the dividing line. Except for station KIA, all the stations lying in the northern region show positive station corrections, and all of those
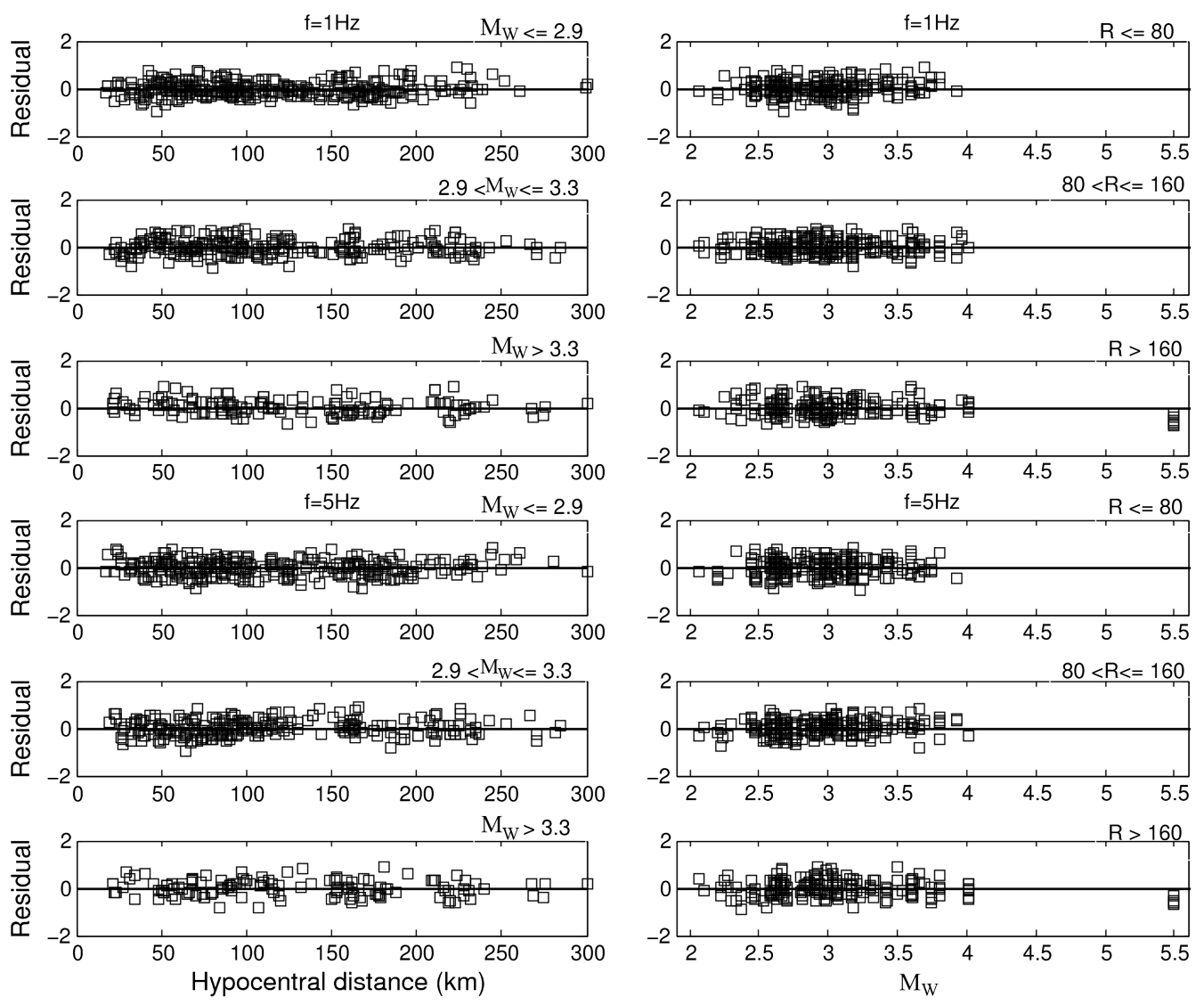

Figure 9. Fourier spectral amplitude residuals versus distance for different magnitude ranges are shown in the left panels. The top left three panels are for $1 \mathrm{~Hz}$ and the bottom left three panels are for $5 \mathrm{~Hz}$. Fourier spectral amplitude residuals versus magnitude for different hypocentral distances are shown in the right panels. The top right three panels are for $1 \mathrm{~Hz}$ and the bottom right three panels are for $5 \mathrm{~Hz}$. $R$ stands for hypocentral distance in $\mathrm{km}$. 


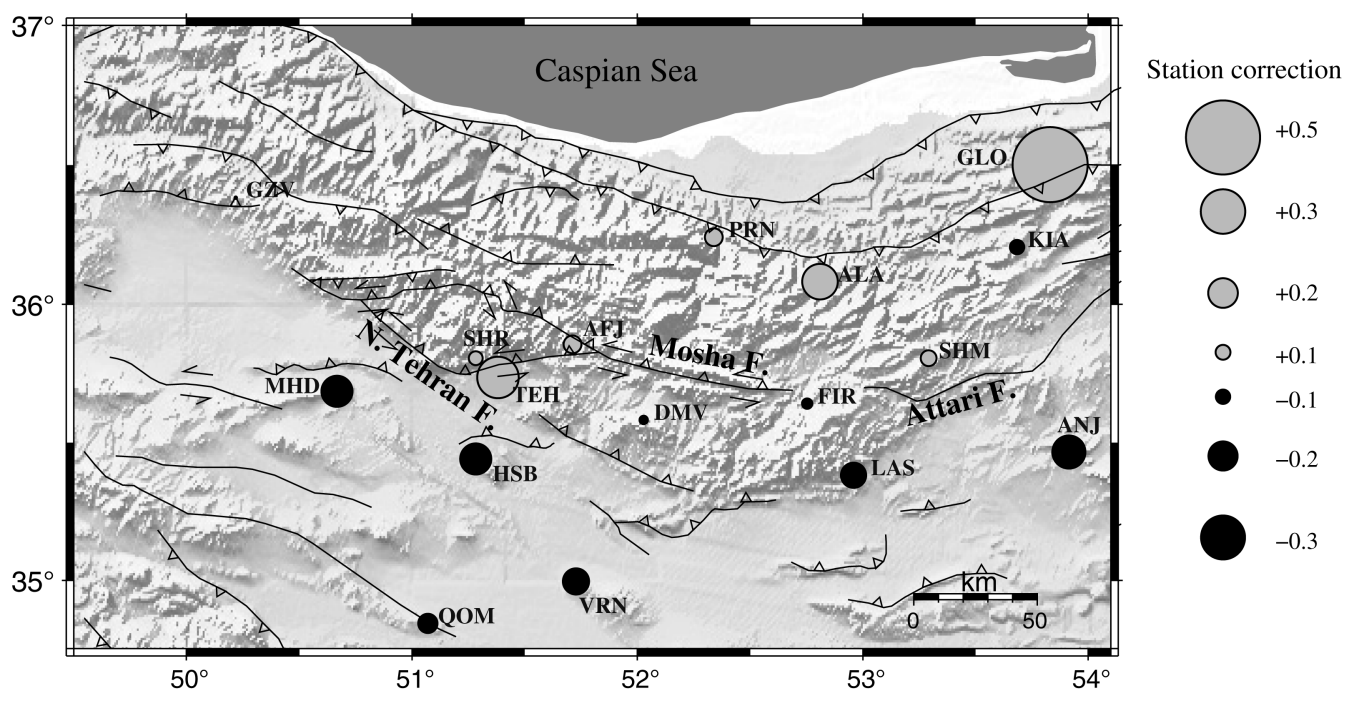

Figure 10. Geographical distribution of amplitude station corrections at $1 \mathrm{~Hz}$. Note that the distribution of the station corrections can be divided along the North Tehran, Mosha, and Attari faults into a positive northern region and a negative southern region.

lying in the southern region show negative station corrections. This implies that attenuation is stronger in the southern region relative to that of the northern region.

Local magnitude station corrections for the mentioned stations (Askari et al., 2009) confirm the these results. The geographical distribution of local magnitude station corrections shows negative and positive station corrections for the northern and southern regions, respectively. Local magnitude station correction is related to the direct $Q$ value around $1 \mathrm{~Hz}$. A positive local magnitude station correction indicates a larger attenuation or smaller $Q$ value. Askari et al. (2009) also calculated a larger attenuation for station KIA, in agreement with our findings. The larger attenuation for station KIA may be related to the fact that it is the only station in our study that is not on hard rock (Ghods and Sobouti, 2005).

Rahimi et al. (2010) did not see significant coda $Q_{0}$ variations across the region. The authors related the observed insignificant variations to differences in the property of the datasets used for the calculation of coda $Q_{0}$ for each station. The contradiction between our results and those of Rahimi et al. (2010) is largely related to differences in the sampling regions of the coda waves and shear waves used in our study. The coda waves used to calculate the coda $Q$ sample a considerable portion of the lithosphere. The shear waves used in this work to calculate the direct $Q$ only sample the crust. Therefore, the observed variation of attenuation across the southern and northern region is related to the lateral variation of attenuation or $Q$ in the crust.

Within the southern region, the absolute value of station corrections is highest for stations very close to the dividing line. The absolute value of the station corrections generally decreases toward the south, away from the dividing line. This may imply that the region along and in the southern vicinity of the dividing line is where the crust is more attenuating.

The larger attenuation of seismic waves for the stations may be related to a change in the path effects (i.e., attenua- tion coefficients) or site effects, or both. The geometrical spreading coefficients may change across the two northern and southern regions due to the possible undulations of the Mohorovičić discontinuity. In order to investigate the possibility, we recalculated the station corrections using rays shorter than $100 \mathrm{~km}$ (Table 1). Only longer rays can be affected by undulations of the Moho depth or variations of intrinsic and scattering attenuation. The station corrections calculated using rays with lengths less than $100 \mathrm{~km}$ show very similar values. This implies that the change in station corrections across the dividing line can be attributed to site effects.

The stations with larger negative station corrections lie on thick Cenozoic-Quaternary rocks with thick interbedded salt and evaporates (National Iranian Oil Company, 1977, 1978), known to have lower seismic velocity and larger attenuation. The stations in the northern region are located either on older Paleozoic rocks or on thick consolidated Tertiary volcanic rocks of the Karaj formation (National Iranian Oil Company, 1977, 1978). Therefore, the variation in the station correction of seismic stations can be linked to the changes in local geology.

\section{Conclusions}

Using earthquake waveforms recorded by local shortperiod IGUT seismic networks, an attenuation relationship for the central Alborz region was derived. By regressing to a hinged trilinear attenuation function with hinges at distances of 80 and $160 \mathrm{~km}$, we found that the geometrical spreading coefficients of $b_{1}=-1.15 \pm 0.21, b_{2}=0.09 \pm$ 0.31 , and $b_{3}=-0.5$ minimize the average absolute value of the Fourier spectrum amplitude residuals. It was found that the dependence of the geometrical spreading coefficients on frequency is insignificant. The reliability of the results was validated by synthetic tests and also the invariability 
of Fourier spectral amplitude residuals with distance and magnitude.

Using the anelastic attenuation coefficient at different frequencies, the direct quality factor $Q$ in the central Alborz region was obtained as $Q=109 \pm 2 f^{0.64 \pm 0.04}$. We showed that the geographical distribution of station corrections at $1 \mathrm{~Hz}$ splits the region along a dividing line connecting the North Tehran, Mosha, and Attari faults into a northern lowattenuating and a southern high-attenuating region. The more attenuating southern region can be linked to the presence of thick Cenozoic-Quaternary sedimentary rocks south of the dividing line.

\section{Data and Resources}

Seismograms used in this study were collected from IGUT. Seismograms can be obtained by writing a formal request to the head of IGUT.

\section{Acknowledgments}

We would like to express our gratitude to IGUT for allowing us to access their earthquake data. We are grateful for the reviews of Dariush Motazedian from Carleton University who greatly improved our manuscript. We wish to thank Associate Editor G. Atkinson, Guoqing Lin, and two other anonymous reviewers for their helpful and constructive comments. We wish to thank H. Rahimi and H. Ghasemi for their useful comments and suggestions. We also thank Eric Bergman of Colorado University at Boulder for improving the writing style of our paper.

\section{References}

Aki, K. (1980). Attenuation of shear waves in the lithosphere for frequencies from 0.05 to $25 \mathrm{~Hz}$, Phys. Earth Planet. In. 21, 50-60, doi 10.1016/ 0031-9201(80)90019-9.

Allen, T. I., P. R. Cummins, T. Dhu, and J. F. Schneider (2007). Attenuation of ground-motion spectral amplitudes in southeastern Australia, Bull. Seismol. Soc. Am. 97, 1279-1292, doi 10.1785/0120060172.

Allen, M. B., M. R. Ghassemi, M. Sharabi, and M. Qoraishi (2003). Accommodation of late Cenozoic oblique shortening in the Alborz range, northern Iran, J. Struct. Geol. 25, 659- 672, doi 10.1016/ S0191-8141(02)00064-0.

Ambraseys, N. N., and C. P. Melville (1982). A history of Persian earthquakes, Cambridge Earth Science SeriesCambridge Univ. Press, London, $240 \mathrm{pp}$.

Askari, R., A. Ghods, and F. Sobouti (2009). Calibration of an $M_{\mathrm{L}}$ scale in the Alborz region, northern Iran, Bull. Seismol. Soc. Am. 99, 268-276, doi 10.1785/0120080122.

Atkinson, G. M. (2004). Empirical attenuation of ground-motion spectral amplitudes in southeastern Canada and the northeastern United States, Bull. Seismol. Soc. Am. 94, 1079-1095, doi 10.1785/0120030175.

Atkinson, G. M., and D. M. Boore (1995). Ground motion relations for eastern North America, Bull. Seismol. Soc. Am. 85, 17-30.

Atkinson, G. M., and R. F. Mereu (1992). The shape of ground motion attenuation curves in southeastern Canada, Bull. Seismol. Soc. Am. 82, 2014-2031.

Berberian, M., and R. S. Yeats (1999). Patterns of historical earthquake rupture in the Iranian plateau, Bull. Seismol. Soc. Am. 89, 120-139.

Bommer, J. J., P. J. Stafford, J. E. Alarcon, and S. Akkar (2007). The influence of magnitude range on empirical ground-motion prediction, Bull. Seismol. Soc. Am. 97, 2152-2170, doi 10.1785/0120070081.
Bondar, I., S. C. Myers, E. R. Engdahl, and E. A. Bergman (2004). Epicentre accuracy based on seismic network criteria, Geophys. J. Int. 156, 483-496, doi 10.1111/j.1365-246X.2004.02070.x.

Boore, D. M. (2003). Simulation of ground motion using the stochastic method, Pure Appl. Geophys. 160, 635-676, doi 10.1007/ PL00012553.

Boore, D. M. (2010). Orientation-independent, nongeometric-mean measures of seismic intensity from two horizontal components of motion, Bull. Seismol. Soc. Am. 100, 1830-1835, doi 10.1785/0120090400.

Boore, D. M., W. B. Joyner, and T. E. Fumal (1997). Equations for estimating horizontal response spectra and peak acceleration from western North American earthquakes: A summary of recent works, Seismol. Res. Lett. 68, 128-153, doi 10.1785/gssrl.68.1.128.

Burger, R., P. Somerville, J. Barker, R. Hermann, and D. Helmberger (1987). The effect of crustal structure on strong ground motion attenuation relations in eastern North America, Bull. Seismol. Soc. Am. 77, 420-439.

Campbell, K. W. (1997). Empirical near-source attenuation relationships for horizontal and vertical components of peak ground acceleration, peak ground velocity, and pseudo-absolute acceleration response spectra, Seismol. Res. Lett. 68, 154-179, doi 10.1785/gssrl.68.1.154.

Campbell, K. W., and Y. Bozorgnia (1994). Empirical analysis of strong ground motion from the 1992 Landers California earthquake, Bull. Seismol. Soc. Am. 84, 573-588.

Cleveland, W. S. (1979). Robust locally weighted regression and smoothing scatterplots, J. Am. Stat. Assoc. 74, 829-836.

Cleveland, W. S., E. Grosse, and W. M. Shyu (1992). Local regression models, in Statistical Models in S, J. M. Chambers and T. J. Hastie (Editors), Wadsworth and Brooks/Cole, Pacific Grove, California, 309-376.

Cormier, V. (1982). The effect of attenuation on seismic body waves, Bull. Seismol. Soc. Am. 72, S169-S200.

Dainty, A. M. (1981). A scattering model to explain seismic $Q$ observations in the lithosphere between 1 and $30 \mathrm{~Hz}$, Geophys. Res. Lett. 8, 11261128, doi 10.1029/GL008i011p01126.

Fletcher, R. (1972). FORTRAN subroutines for minimization by quasiNewton methods, United Kingdom Atomic Energy Authority Report AERE R7125 Atomic Energy Research Establishment, Harwell, England, $36 \mathrm{pp}$.

Frankel, A. (1991). Mechanism of seismic attenuation in the crust: Scattering and anelasticity in New York State, South Africa, and southern California, J. Geophys. Res. 96, 6269-6289, doi 10.1029/91JB00192.

Gagnepain-Beyneix, J. (1987). Evidence of spatial variations of attenuation in the western Pyrenean range, Geophys. J. Roy. Astron. Soc. 89, 681704, doi 10.1111/j.1365-246X.1987.tb05187.x.

Ghasemi, H., M. Zare, Y. Fukushima, and K. Koketsu (2009). An empirical spectral ground-motion model for Iran, J. Seismol. 13, 499-515, doi 10.1007/s10950-008-9143-x.

Ghods, A., and F. Sobouti (2005). Quality assessment of seismic recording: Tehran seismic telemetry network, J. Asian Earth Sci. 25, 687-694, doi 10.1016/j.jseaes.2004.08.001.

Hanks, T. C., and H. Kanamori (1979). A moment magnitude scale, J. Geophys. Res. 84, 2348-2350, doi 10.1029/JB084iB05p02348.

Hartzell, S. (1978). Earthquake aftershocks as Green's functions, Geophys. Res. Lett. 5, 1-4, doi 10.1029/GL005i001p00001.

Hessami, K., F. Jamali, and H. Tabassi (2003). Major active faults of Iran, Ministry of Science, Research, and Technology, International Institute of Earthquake Engineering and Seismology, scale 1:2,500,000.

Jackson, J., K. Priestley, M. Allen, and M. Berberian (2002). Active tectonics of the South Caspian Basin, Geophys. J. Int. 148, 214 245, doi 10.1046/j.1365-246X.2002.01588.x.

Kanamori, H. (1977). The energy release in great earthquakes, J. Geophys. Res. 82, 2981-2987, doi 10.1029/JB082i020p02981.

Lussou, P., Y. Fukushima, P. Y. Bard, and F. Cotton (2001). Seismic design regulation codes: Contribution of K-Net data to site effect evaluation, J. Earthq. Eng. 5, 13-33, doi 10.1080/13632460109350384.

Massa, M., P. Morasca, S. Marzorati, G. Costa, and D. Spallarossa (2008). Empirical ground-motion prediction equation for northern Italy using 
weak- and strong-motion amplitudes, frequency content, and duration parameters, Bull. Seismol. Soc. Am. 98, 1319-1342, doi 10.1785/ 0120070164.

Meirova, T., R. Hofstetter, A. Ben-Avraham, D. M. Steinberg, L. Malagnini, and A. Akicnci (2008). Weak-motion-based attenuation relationship for Israel, Geophys. J. Int. 175, 1127-1140, doi 10.1111/j.1365246X.2008.03953.x.

Motazedian, D. (2006). Region-specific key seismic parameters for earthquakes in northern Iran, Bull. Seismol. Soc. Am. 96, 1383-1395, doi $10.1785 / 0120050162$.

National Iranian Oil Company (NIOC) (1977). Geological map of Iran sheet 2: North-Central Iran, scale 1:1, 000,000.

National Iranian Oil Company (NIOC) (1978). Geological map of Iran sheet 1: North-West Iran, scale 1:1,000,000.

Ou, G., and R. Herrmann (1990). A statistical model for ground motion produced by earthquakes at local and regional distances, Bull. Seismol. Soc. Am. 80, 1397-1417.

Rahimi, H., K. Motaghi, S. Mukhopadhyay, and H. Hamzehloo (2010). Variation of coda wave attenuation in the Alborz region and central Iran, Geophys. J. Int. 181, 1643-1654, doi 10.1111/j.1365246X.2010.04574.x.
Raoof, M., R. Herrmann, and L. Malagnini (1999). Attenuation and excitation of three-component ground-motion in southern California, Bull. Seismol. Soc. Am. 89, 888-902.

Steidl, J. H., A. G. Tumarkin, and R. J. Archuleta (1996). What is a reference site? Bull. Seismol. Soc. Am. 86, 1733-1748.

Wilkie, J., and G. Gibson (1995). Estimation of seismic quality factor $Q$ for Victoria, Australia, AGSO J. Geol. Geophys. 15, 511-517.

Zare, M. (1999). Conribution à létude des mouvements forts en Iran: Du catalogue aux lois d’atténuation, Thése de Doctorat, Université de Grenoble at Saint-Martin-d'Hères, Franc (in French).

Zare, M., and S. Sabzali (2006). Spectral attenuation of strong motions in Iran, in Proc. of the 3rd International Symposium on the Effects of Surface Geology on Seismic Motion, Grenoble, France, 30 August1 September 2006.

Institute for Advanced Studies in Basic Sciences (IASBS)

Zanjan, Iran

Manuscript received 30 November 2010 


\section{Queries}

1. I revised "...using a local regression smoothing method called Robust LOWESS (locally weighted scatterplot smoothing)" to "...using a locally weighted scatterplot smoothing (LOWESS, local regression smoothing method) called robust LOWESS." This was done to place the acronym immediately after the term that it stands for at its first usage in the abstract and in the main text, per BSSA guidelines. Please advise whether your meaning is retained.

2. Please indicate the meaning of HRV as to whether it is the name of a seismic event, seismic station etc.

3. I tentatively revised "...for the intervals of $80-120$ and $120-160 . .$. " to "...for the intervals of $80-120 \mathrm{~km}$ and 120 $160 \mathrm{~km}$...” Please advise whether your meaning is retained. 